\title{
Inbreeding depression and low between-population heterosis in recently diverged experimental populations of a selfing species
}

\author{
Y Rousselle, M Thomas, N Galic, I Bonnin ${ }^{1}$ and I Goldringer \\ UMR Génétique Végétale, INRA-CNRS-Univ Paris-Sud-AgroParisTech, Ferme du Moulon, Gif sur Yvette, France
}

\begin{abstract}
In fragmented populations, genetic drift and selection reduce genetic diversity, which in turn results in a loss of fitness or in a loss of evolvability. Genetic rescue, that is, controlled input of diversity from distant populations, may restore evolutionary potential, whereas outbreeding depression might counteract the positive effect of this strategy. We carried out self-pollination and crosses within and between populations in an experimental subdivided population of a selfing species, Triticum aestivum L., to estimate the magnitude of these two phenomena. Surprisingly, for a self-fertilizing species, we found significant inbreeding depression within each population for four of the six traits studied, indicating that mildly deleterious mutations were still segregating in these populations. The progeny of within- and betweenpopulation crosses was very similar, indicating low between-
\end{abstract}

population heterosis and little outbreeding depression. We conclude that relatively large population effective sizes prevented fixation of a high genetic load and that local adaptation was limited in these recently diverged populations. The kinship coefficient estimated between the parents using 20 neutral markers was a poor predictor of the progeny phenotypic values, indicating that there was a weak link between neutral diversity and genes controlling fitnessrelated traits. These results show that when assessing the viability of natural populations and the need for genetic rescue, the use of neutral markers should be complemented with information about the presence of local adaptation in the subdivided population.

Heredity (2011) 106, 289-299; doi:10.1038/hdy.2010.72; published online 9 June 2010

Keywords: inbreeding depression; outbreeding depression; local adaptation; genetic rescue; genetic load; heterosis

\section{Introduction}

Dynamic management (DM) is an evolutionary approach for the conservation of the genetic resources of crop species (Henry et al., 1991; Goldringer et al., 2001). DM aims at maintaining the genetic diversity of crop species by growing successive generations of genetically diverse populations in several contrasted environments, without intentional human selection. Similar to wild fragmented populations, the mid-term maintenance of the evolutionary potential of the DM populations is a major issue for the sustainable conservation of biodiversity. In isolated populations, drift and selection lead to the reduction of genetic diversity. Reduced genetic diversity might then result in a loss of fitness because of inbreeding depression caused by the accumulation of deleterious mutations (Lynch et al., 1995; Keller and Waller, 2002) and a loss of evolvability, that is, the ability of a population to respond to natural or artificial selection (Houle, 1992). Isolation is not the only threat

Correspondence: $\operatorname{Dr}$ Y Rousselle, UMR Génétique Végétale, INRACNRS-Univ Paris-Sud-AgroParisTech, Ferme du Moulon, Gif sur Yvette 91190, France.

E-mail: yves.rousselle@moulon.inra.fr

${ }^{1}$ Current address: Fondation pour la Recherche sur la Biodiversité (FRB), 57 rue Cuvier, CP 41, 75231 Paris Cedex 05, France.

Received 23 November 2009; revised 23 April 2010; accepted 5 May 2010; published online 9 June 2010 for natural populations. In the case of connected populations, genetic load seems to be more important in very small populations, as shown theoretically by Theodorou and Couvet (2006) and by crossing experiments by Willi and Fischer (2005).

Introduction of migrants in the threatened populations may allow the restoration of evolutionary potential by introducing new alleles into the population and hence (1) increasing the within-population genetic variability and (2) producing heterosis by masking deleterious recessive mutations or increasing the level of heterozygosity. The potential positive effect of migration to manage natural fragmented populations has often been discussed in the literature (reviewed in Tallmon et al., 2004), but few studies have really applied the so-called 'genetic rescue' to real populations and surveyed its consequences. Madsen et al. (1999) applied it to inbred populations of adder species and Vila (2003) applied genetic rescue to Scandinavian wolf populations. In both cases, the target population size increased very quickly, in one or two generations, despite the very few number of migrants used (20 and 1, respectively). Similarly, in plants, Newman and Tallmon (2001) showed that migration increased the fitness of experimental populations of Brassica campestris.

Real application of genetic rescue in conservation strategies is infrequent, probably because of the risk of outbreeding depression. Outbreeding depression is the 
reduction in fitness of offspring from matings between genetically divergent individuals. Experimental crosses between populations are the most frequent way of measuring the amount of outbreeding depression in a structured population (Edmands, 2007). Several experimental crossing studies have been carried out on fragmented populations and have revealed either outbreeding depression (Fischer and Matthies, 1997; Waser et al., 2000; Willi et al., 2005; Galloway and Etterson, 2005), heterosis between populations (Fenster and Galloway, 2000; Bossuyt, 2007) or both within one species (Physa acuta; Escobar et al., 2008).

In general, two non-exclusive mechanisms may explain outbreeding depression (Schierup and Christiansen, 1996): (1) the 'ecological mechanism', which is also called the 'dilution effect', in which, due to local adaptation of the populations, individuals carrying migrant genes have a lower fitness because these genes are maladapted to the new environment (Waser, 1993) and (2) the 'genetic mechanism' in which positive epistatic interactions within gene complexes might be broken (Lynch, 1991), whereas deleterious epistatic interactions appear. The two mechanisms may have different influences on the different generations of the progeny after the crossing event, depending on the genetic architecture of fitness traits (for example, dominance) and on the mating system. In outcrossing species in which heterozygosity is high, complexes of genes could be selected by dominance-by-additive epistasis. The dominance component of these complexes might be broken during the first generation, leading to outbreeding depression because of the genetic mechanism (Escobar et al., 2008). On the contrary, in selfing species, in which homozygosity is high, mainly additive-byadditive epistasis is expected to be found (Goldringer et al., 1997). In the first generation after a cross, additiveby-additive epistasis complexes are not broken and hence epistasis is not expected to contribute much to outbreeding depression in selfing species. However, in selfing species underdominance might also contribute to outbreeding depression of the first generation. Under both mating systems, the first generation is expected to be further depressed by the ecological mechanism, that is, the additive effects of the migrant maladapted alleles, except in the rare case in which the migrant alleles are fully recessive. Although the ecological mechanism persists after the second generation, genetic incompatibility is expected to be fully expressed as recombination breaks cis parental genes complexes (Lynch, 1991). Other mechanisms could also be involved in outbreeding depression, such as interactions between Wolbachia and a fig wasp (Greeff et al., 2009).

When applying genetic rescue approaches, two components of migration should be studied: the 'quality' of the migrants and the quantity of the migrants. Managing the quantity of migrants is well described by Wang (2004), who applied the One-Migrant-per-Generation rule to conservation biology. We will not discuss this component of the genetic rescue but will focus on the 'quality' component. Migrant origin should be carefully chosen to benefit from the renewal of genetic variability while limiting outbreeding depression effects. A major issue is to identify the appropriate distance (geographical or genetic) of the migrant individuals. This distance can be described as the optimal crossing distance, and it depends mainly on the genetic structure of the populations, on the importance of local adaptation and on the mating system (Waser, 1993). Most of the studies described above estimate divergence between populations by geographical distances. A few estimate differentiation at the genetic level by kinship coefficient (Willi et al., 2005) or by $\mathrm{F}_{\mathrm{ST}}$ (Escobar et al., 2008) or at the phenotypic level by $Q_{\mathrm{ST}}$ (Escobar et al., 2008).

In this study, we investigated the conditions to set up a genetic rescue approach in an experimental DM system of a self-fertilizing crop species, bread wheat (Triticum aestivum L.). To this aim, genetic diversity and phenotypic variability were analyzed in three DM populations after 12 generations of evolution without conscious human selection. Previous studies on these populations have shown that populations have diverged for different traits, such as disease resistance (Paillard et al., 2000) and earliness (Goldringer et al., 2006), in relation to local conditions. Three levels of crosses (selfing and withinpopulation and between-population crosses) were carried out and phenotypic values of the progeny were analyzed to assess individual inbreeding depression and between-population heterosis or outbreeding depression. Kinship coefficients between the parents of the crosses were estimated using molecular markers, and the relation between the kinship coefficient between parents and the progeny values was analyzed to test for the existence of an optimal genetic distance. These results, together with analyses of the genetic structure of the populations, allowed to improve our understanding of the DM system and to make decisions regarding migration strategies.

As the DM system corresponds to recently diverged populations, the findings of our study can be useful not only for designing optimized dynamic management of genetic resources for crops, but also for improving conservation strategies for wild species experiencing recent habitat fragmentation.

\section{Materials and methods}

\section{Plant material}

Development of the dynamic management populations: A total of 16 nearly homozygous genotypes (referred to as 'initial lines' hereafter) of Triticum aestivum L. representing a wide genetic basis were initially crossed using a pyramidal design (Goldringer et al., 2006). The hybrid seeds obtained were then sown and bulk harvested for three generations of seed multiplication. This composite cross population, G0, was used as the initial population of the DM system. In 1984, seed samples of G0 were distributed to three sites of a French experimental network, Le Moulon $\left(48.4^{\circ} \mathrm{N}, 2.1^{\circ} \mathrm{E}\right)$, Rennes $\left(48.1^{\circ} \mathrm{N}\right.$, $\left.1.4^{\circ} \mathrm{W}\right)$ and Toulouse $\left(43.4^{\circ} \mathrm{N}, 1.3^{\circ} \mathrm{E}\right)$, with contrasted environmental conditions. Since then, each population has been cultivated every year without any conscious human selection. Each year, the whole population was harvested in bulk, from which a random sample of at least 5000 seeds was sown in a $100 \mathrm{~m}^{2}$ plot isolated from other wheat cultures to constitute the next generation. Migration between populations has been avoided since the beginning (see Goldringer et al., 2006; Rhoné et al., 2008 for more details on the populations). 
Crossing scheme: Because wheat plants are hermaphrodites, plants were considered either as females and the spikes for crosses were emasculated or as males and only their pollen were used. In each of the three populations of the twelfth generation (Le Moulon, Rennes and Toulouse), 30-39 female plants and 35-38 male plants were randomly chosen for the crossing scheme. For each female plant, three types of crosses were made (Figure 1): self-pollination (SELF), withinpopulation crosses (WPC, two distinct samples were used for female plants and male plants in each population) and between-population crosses (BPC, cross between a female from one population and a male from each of the two other populations). The male sample in each population was the same for the two last types of crossing (WPC and BPC). For each cross, different spikes of the female plant were either selfed or pollinated during spring 2003 and harvested during summer 2003. The progeny from the resulting seeds of each spike was called a family. The total number of families was 416: 104 for the SELF type, 104 for the WPC type and 208 for the BPC type.

\section{Phenotypic evaluation}

The first-generation progeny of all the crosses were sown in a common-garden experiment at Le Moulon in October 2006 in a two-replicate randomized complete block design. In each block, each family was evaluated with a $1.20 \mathrm{~m}$ single row of 15 seeds. The first replicate was cultivated without fungicide and phenotypic measures were recorded on each plant of each row. The second replicate was cultivated with fungicide to ensure seed quality at harvest and only average phenotypic values were recorded for each row.

Six phenotypic traits, related to plant fitness, were measured at maturity during the harvest in summer 2007: plant height (PH), spike number per plant (SNP), kernel number per plant (KNP), kernel number per spike

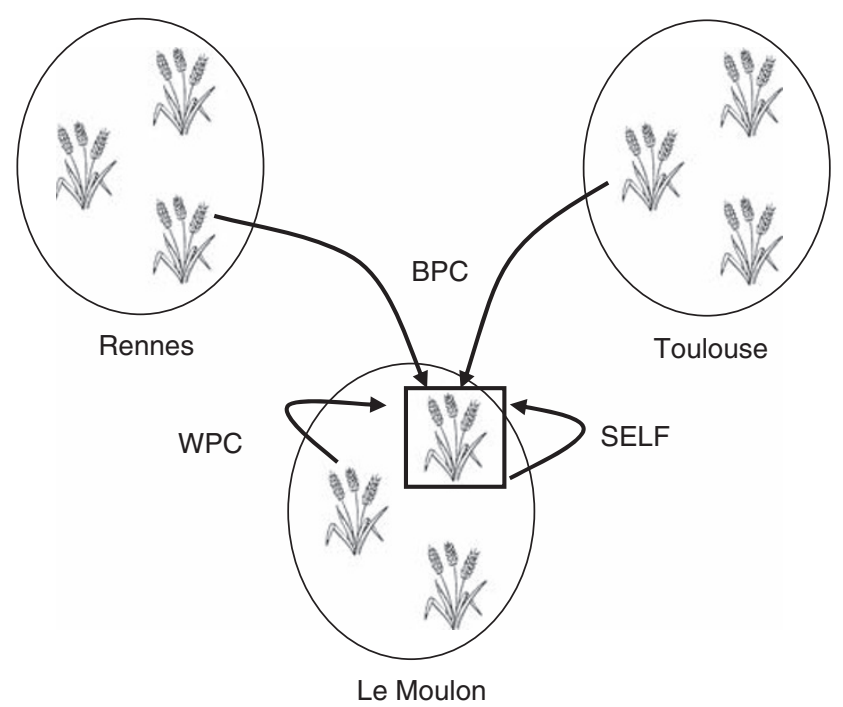

Figure 1 Crossing scheme. Each ellipse represents a population (Le Moulon, Rennes and Toulouse). Cross type codes are SELF for selfing, WPC for within-population cross and BPC for betweenpopulation cross. The framed plant is the target female plant for the four crosses symbolized by the arrows.
(KNS), grain weight per plant (GWP) and thousandkernel weight (TKW).

Genotyping of the parents of the crosses

In spring 2003, a leaf sample was taken on each parent used in the crossing scheme for genotyping. The 16 initial lines of the DM populations were sown in 2007 and they were also sampled for genotyping. For each plant, total DNA was extracted from $100 \mathrm{mg}$ of fresh material following a protocol derived from the Dneasy 96 Plant Kit (Qiagen, Hilden, Germany).

In all, 17 microsatellite markers developed by Röder et al. (1998), 1 (wmc231) by Somers and Edwards (2004) and a bi-loci marker (the pair of primers amplifies two individual loci on 2 chromosomes) (cfd71) developed by Guyomarc'h et al. (2002) were used for genotyping the 217 individuals studied. This set of 20 markers covers the 21 chromosomes of bread wheat except the chromosome 1A. PCR protocols were adapted from Röder et al. (1998) and Guyomarc'h et al. (2002). Amplified fragments were separated on an ABI 3130xl semi-automatic sequencer (Applied Biosystems, Foster City, CA, USA) and analyzed using GeneMapper 3.7 (Applied Biosystems).

\section{Analyses}

Genetic diversity among parents: We analyzed the genetic diversity of the individuals involved in the crossing scheme and of the 16 parents of the DM populations. Sample sizes for each population (Le Moulon, Rennes and Toulouse) were 67, 70 and 76, respectively. For each population, gene diversity was estimated from the allelic frequencies using the unbiased estimator $H_{\mathrm{e}}$ (Nei, 1987). Mean average over loci of allele number per locus (A) and within-population fixation index $\mathrm{F}_{\text {IS }}$ were computed using Genetix 4.03 (Belkhir et al., 2000, Montpellier, France). The $95 \%$ confidence interval of $F_{\text {IS }}$ was computed using 1000 bootstraps per locus. Differentiation between the three populations was estimated using Weir and Cockerham F-statistics estimator $\theta$ (Weir and Cockerham, 1984) with 1000 permutations used for significance tests. We also estimated differentiation between the three populations by the $D_{\mathrm{EST}}$ measure (Jost, 2008). In contrast to $\mathrm{F}_{\mathrm{ST}}$ estimator $\theta, D_{\mathrm{EST}}$ partitions total genetic variance into statistically independent within- and between-population components and therefore avoids underestimation of differentiation by $\mathrm{F}_{\mathrm{ST}}$-like measures because of high within-population diversity (Jost, 2008). We estimated population genetic effective sizes $\left(\hat{N}_{\mathrm{e}}\right)$ between the 16 initial lines and the populations at the twelfth generation using temporal variation in allele frequencies estimated by the mean allelic frequency standardized variance $\left(F_{c}\right.$, Nei and Tajima, 1981). We used the second sampling scheme from Waples (1989). We estimated $95 \%$ confidence intervals assuming that $\left(n \hat{F}_{c}\right) / E\left(\hat{F}_{c}\right)$ follows a $\chi^{2}$ with $n$ degree of freedom (total number of alleles). We compared the genetic effective sizes obtained with the demographic sizes corrected for selfing following: $N_{\text {selfing }}=\left(N_{\text {demographic }}\right) /\left(1+\mathrm{F}_{\text {IS }}\right)$ (Pollak, 1987).

To investigate the possibility for an optimal genetic distance for crossing, we computed kinship coefficient for each pair used in the crossing scheme using SPAGeDi (Hardy and Vekemans, 2002, Bruxelles, Belgique), a software program that implements the computation of the kinship coefficient proposed by Loiselle et al. (1995). 
Statistical analysis of quantitative traits

Preliminary analysis and correction for spatial effects: In the first replicate, we thought that the position of the plants in the row might influence trait expressions because of differences in light exposure such as, for instance, border effects for plants at the start and end position. To account for such effects, we tested a 'position' effect using the following analysis of variance (ANOVA) model that explained phenotypic traits measured on the first replicate: $Y_{i j}=\mu+\operatorname{pos}_{i}+$ Fam $_{j}+R_{i j}$ where pos is the fixed position effect, Fam is the random family effect and $R$ is the random residual. Family and position effects were significant for all the traits, and hence we corrected each individual trait value in the first replicate for the position effect. In the following analyses of quantitative traits, means of corrected individual values were used for each family in the first replicate. Thus, for each trait, each family was described by two values, one for each replicate. The estimated broad-sense heritability at the design level ranged from 0.25 to 0.86 , indicating the high level of genetic variation and the high correlation between the two replicates, although the experimental conditions of the two replicates differed.

Genetic structure at quantitative traits: To describe the genetic structure in parental populations for the quantitative traits, we used the selfed progeny (32 families for Le Moulon, 34 for Rennes and 38 for Toulouse) to assess the $Q_{\mathrm{ST}}$ statistics (Wright, 1969; Spitze, 1993) as follows:

$$
Q_{\mathrm{ST}}=\frac{\left(\hat{\sigma}_{B}^{2}\left(1+\mathrm{F}_{I S}\right)\right)}{\left(2 \hat{\sigma}_{W}^{2}+\hat{\sigma}_{B}^{2}\left(1+\mathrm{F}_{I S}\right)\right)}
$$

where $\hat{\sigma}_{B}^{2}$ is the estimated between-population genetic variance, $\hat{\sigma}_{W}^{2}$ is the estimated within-population genetic variance and $F_{I S}$ is the Wright fixation index. The withinand the between-population genetic variances in the experiment were estimated with the following ANOVA model:

$$
Y_{i j k}=\mu+\operatorname{Pop}_{i}+\operatorname{Fam}(\operatorname{Pop})_{i j}+\operatorname{rep}_{k}+R_{i j k}
$$

where Pop is the random population effect, Fam is the random family effect, rep is the fixed replicate effect and $R$ is the random residual. $\hat{\sigma}_{B}^{2}$ was estimated as the variance associated with the Pop effect and $\hat{\sigma}_{W}^{2}$ as the variance associated with Fam. $Q_{\mathrm{ST}}$ was computed when the Pop effect was significant.

Sources of variation for the progeny phenotypic values: Using all progeny (104 SELF, 104 WPC and 208 BPC), we tested the effect of cross type and the effect of the population of origin of the female plant with the following ANOVA model:

$$
\begin{aligned}
Y_{i j k l}= & \mu+\text { ctype }_{i}+\text { OriginFpl }_{j}+(\text { ctype } \times \text { OriginFpl })_{i j} \\
& + \text { Fam }(\text { ctype } \times \text { OriginFpl })_{i j k}+\operatorname{rep}_{l}+R_{i j k l}
\end{aligned}
$$

where ctype is the fixed effect of cross type as defined in the crossing scheme (SELF, WPC and BPC), OriginFpl is the random effect of the population of origin of the female plant (Le Moulon, Rennes and Toulouse) and the other effects are the same as described in model (1).

To analyze how the relatedness between the parents of a cross affected the phenotypic value of the progeny, the last model included the kinship coefficient $K$ as covariates as follows:

$$
\begin{aligned}
Y_{i j}= & \mu+\text { OriginFpl }_{i}+\alpha K+\beta K^{2}+\alpha_{i} K+\beta_{i} K^{2} \\
& +\operatorname{rep}_{j}+R_{i j}
\end{aligned}
$$

We included the square of the kinship coefficient $\left(K^{2}\right)$ to define the phenotypic value of the progeny as a parabolic function of the kinship coefficient. A significant $K^{2}$ effect would indicate that there is an optimal kinship coefficient maximizing the progeny value.

To test for local adaptation, we have compared leastsquare means of WPC progeny from the Le Moulon population (considered as residents as experimentation took place in Le Moulon), with Rennes or Toulouse WPC progeny (considered as migrants) and corresponding BPC (Le Moulon by Rennes and Le Moulon by Toulouse in both direction). Least-square means were estimated using the following model:

$$
Y_{i j k l}=\operatorname{cross}_{i}+\operatorname{Fam}(\text { cross })_{i j}+\operatorname{rep}_{k}+R_{i j k l}
$$

where cross is the fixed effect of the cross type taking into account the origin of the two parents and the other effects are the same as described in model (1).

All ANOVA analyses were run using PROC generalized linear model (GLM) in SAS (SAS Institute, 2000, Carry, NC, USA).

Hierarchical heterosis estimation: Finally, applying model (4) to the progeny of all crosses, we estimated heterosis at two levels: (1) within population and (2) between populations. For (1), we estimated withinheterosis for each population (Le Moulon, Rennes and Toulouse) as $\left(Y_{\mathrm{WPC}, i}-Y_{\mathrm{SELF}, i}\right) /\left(Y_{\mathrm{WPC}, i}\right)$ where $Y_{\mathrm{WPC}, i}$ and $Y_{\mathrm{SELF}, i}$ are, respectively, the mean trait value for the progeny from within-population crosses and from selfing in population $i$. For (2), we estimated betweenheterosis for each pair of populations (Le Moulon-Rennes, Le Moulon-Toulouse and Rennes-Toulouse) as

$$
\frac{\left(Y_{\mathrm{BPC}, i j}-\left(\left(Y_{\mathrm{WPC}, i}+Y_{\mathrm{WPC}, j}\right) / 2\right)\right)}{Y_{\mathrm{BPC}, i j}}
$$

where $Y_{\mathrm{BPC}, i j}$ is the mean trait value for progeny from crosses between population $i$ and population $j$ for both directions. To test whether heterosis values are significantly different from 0 , we applied a $t$-test on heterosis numerator with the ESTIMATE statement of generalized linear model (GLM) procedure in SAS.

\section{Results}

\section{Population structure}

Table 1 shows diversity statistics for the 16 initial lines of the DM system and for the three parental populations. Although the Rennes population had a slightly lower $H_{\mathrm{e}}$, the values for the Nei diversity were quite close in the populations of the twelfth generation and in the 16 initial lines. This result indicated that there was no marked loss of within-population diversity during the 12 generations of DM. This was confirmed by the allele number. A factorial component analysis showed that the initial diversity of the 16 lines was included in the total diversity of the three populations (data not shown). For all populations, the fixation index was rather close to 1 , from 0.84 (Rennes population) to 0.98 (16 initial lines), 
Table 1 Diversity statistics for the three parental populations and for the 16 initial lines of the DM system

\begin{tabular}{|c|c|c|c|c|c|}
\hline & $\mathrm{n}$ & $A$ & $\mathrm{H}_{e}$ (s.e.) & $F_{I S}$ & $\mathrm{~N}_{e}(95 \% C I)$ \\
\hline 16 initial lines & 16 & 4.0 & $0.61(0.16)$ & 0.98 & - \\
\hline Le Moulon population & 67 & 5.0 & $0.61(0.18)$ & 0.93 & $173(120-244)$ \\
\hline Rennes population & 70 & 3.9 & $0.51(0.18)$ & 0.84 & $67(46-93)$ \\
\hline Toulouse population & 76 & 5.0 & $0.59(0.15)$ & 0.97 & $95(68-128)$ \\
\hline
\end{tabular}

Abbreviations: A, mean allele number; DM, dynamic management; $\mathrm{F}_{\mathrm{IS}}$, fixation index; $H_{\mathrm{e}}$, Nei diversity; $n$, sample size; $N_{\mathrm{e}}$, effective size.

and significantly different from zero, as expected for a highly selfing species such as bread wheat. Genetic effective sizes ranged from 67 (95\% confidence interval 46-93) for Rennes population to 173 (95\% confidence interval 120-244) for Le Moulon population. They were consistent with the within-population diversity $H_{\mathrm{e}}$ : Rennes population had the smallest value $\left(H_{\mathrm{e}}=0.51\right)$ and Le Moulon population the highest $\left(H_{\mathrm{e}}=0.61\right) . N_{\mathrm{e}}$ values were 15-39 times smaller than demographic sizes corrected for selfing, that is, 2650.

Global differentiation over the three populations $\left(\theta=0.07\right.$ and $\left.D_{\mathrm{EST}}=0.11\right)$ was significantly different from zero (tests performed on $\theta$ only). Differentiation $(\theta)$ for each pair of populations ranged from 0.05 ( 0.09 for $D_{\text {EST }}$ ) between populations from Toulouse and Le Moulon to 0.10 ( 0.14 for $\left.D_{\text {EST }}\right)$ between populations from Toulouse and Rennes, with an intermediate value of 0.07 ( 0.10 for $D_{\text {EST }}$ ) between populations from Le Moulon and Rennes. The three values of $\theta$ were significantly different from zero, indicating spatial differentiation in the DM system. $D_{\mathrm{EST}}$ values were of the same order than $\theta$ while slightly higher. In addition, $D_{\mathrm{EST}}$ ranked pairs of populations as $\theta$.

In model (1), the population effect was significant for PH, KNS and GWP. $Q_{\text {ST }}$ values for these traits were 0.29, 0.19 and 0.19 , respectively, whereas $\mathrm{F}_{\mathrm{ST}}$ was 0.07 (Table 2). Therefore, differentiation for these quantitative traits was strong, as illustrated by the ratio $Q_{\mathrm{ST}} / \mathrm{F}_{\mathrm{ST}}$ equal to 4.14 , 2.71 and 2.71, respectively. Although $D_{\text {EST }}(0.11)$ was higher than $\theta$, quantitative differentiation was still higher than neutral differentiation. Plant height differentiation was mainly because of the higher mean value for Rennes progeny (Table 2), whereas Toulouse progeny had significantly lower mean GWP and KNS, leading to significant $Q_{\mathrm{ST}}$ for these two traits.

\section{Sources of variation for progeny phenotypic values}

Model (2) showed significant cross type and family effects for all traits (Table 3). Progeny derived from selfing had significantly lower values than the progeny issued from the two other cross types for KNP, GWP, KNS and TKW, indicating marked individual inbreeding depression (Table 4). For PH and SNP, progeny from selfing had significantly lower mean values than between-population cross progeny and lower but not significantly different mean values than within-population cross progeny. Mean values for traits for progeny from between-population crosses were never significantly different than those of progeny from withinpopulation crosses. The effect of the population of origin of the female plant was significant for all traits except for SNP and KNP. These results were consistent with the high $Q_{\mathrm{ST}}$ values estimated for PH, KNS and GWP on selfed progeny, whereas $Q_{\mathrm{ST}}$ was not significant for TKW. Progeny issued from female plants of the Rennes
Table 2 Quantitative differentiation among selfed progeny

\begin{tabular}{lcccl}
\hline \multirow{2}{*}{ Traits } & \multicolumn{2}{c}{ Least-square means for the Pop effect } & \multirow{2}{*}{$\mathrm{Q}_{S T}$} \\
\cline { 2 - 4 } & Le Moulon & Rennes & Toulouse & \\
\hline PH & $106.27(\mathrm{~A})$ & $117.89(\mathrm{~B})$ & $103.89(\mathrm{~A})$ & $0.29^{* * *}$ \\
SNP & 11.03 & 10.72 & 10.95 & 0 (NS) \\
KNP & 472.10 & 481.75 & 443.52 & $0.1(\mathrm{NS})$ \\
GWP & $20.98(\mathrm{~A})$ & $22.24(\mathrm{~A})$ & $19.77(\mathrm{~B})$ & $0.19^{*}$ \\
KNP & $42.44(\mathrm{~A})$ & $44.43(\mathrm{~A})$ & $40.24(\mathrm{~B})$ & $0.19^{*}$ \\
TKW & 44.16 & 45.55 & 44.26 & 0.05 (NS)
\end{tabular}

Abbreviations: GWP, grain weight per plant; KNP, kernel number per plant; KNS, kernel number per spike; NS, non significant; $\mathrm{PH}$, plant height; SNP, spike number per plant; TKW, thousandkernel weight.

Capital letters near the least-square means correspond to groupings from pairwise multiple comparison between the populations of origin. $Q_{\mathrm{ST}}$ for all the traits.

${ }^{*} P$-value $<0.05,{ }^{* * *} P$-value $<0.0001$.

population were significantly taller and had higher TKW and GWP (Table 5). Progeny derived from female plants of the Toulouse population had a significantly lower KNS. The interaction between cross type and population of origin of the female plant was not significant for any trait except $\mathrm{PH}$.

The kinship coefficient $K$ had a significant effect on $\mathrm{PH}$ and GWP and a significant interaction effect with the female plant origin for TKW. $K^{2}$ also had a significant effect on TKW in interaction with the female plant origin. Despite these significant effects of $K$, model (3) explained only $4-23 \%$ of the phenotypic variation compared with 59-88\% explained by model (2). The discrete ctype effect explained much more phenotypic variation than the continuous $K$ covariate.

The cross effect of model (4) was significant only for PH, SNP and TKW. For these three traits, phenotypic values of Le Moulon WPC progeny were significantly lower than PH of Rennes WPC progeny but not different than PH of Toulouse WPC progeny (see Figure 2). Reciprocal between-population crosses did not show any asymmetry except for PH between the Toulouse population and the Le Moulon population. In this case, BPC progeny from a female plant from the Le Moulon population had a significantly lower PH than BPC progeny from male plants from the Le Moulon population. We found no indication of a specific maternal effect.

Within-population heterosis was much greater than between-population heterosis for all the quantitative traits (Figure 3). KNP and GWP showed large and significant heterosis in the Toulouse population ( 0.17 and 0.21 , respectively) and in the Rennes population $(0.12$ and 0.17, respectively), whereas heterosis was not significant in the Le Moulon population (0.06 and 0.10, 
Table 3 ANOVA table with model (2)

\begin{tabular}{|c|c|c|c|c|c|c|c|c|c|c|c|c|c|}
\hline \multirow[t]{2}{*}{ Source of variation } & \multirow[t]{2}{*}{ d.f. } & \multicolumn{2}{|c|}{ PH } & \multicolumn{2}{|c|}{ SNP } & \multicolumn{2}{|c|}{ KNP } & \multicolumn{2}{|c|}{$G W P$} & \multicolumn{2}{|c|}{ KNS } & \multicolumn{2}{|c|}{$T K W$} \\
\hline & & SS & F-value & SS & F-value & SS & F-value & SS & F-value & SS & F-value & SS & F-value \\
\hline ctype & 2 & 2112 & $4.93^{* *}$ & 67 & $4.57^{*}$ & 662066 & $16.14^{* * *}$ & 2921 & $30.57^{* * *}$ & 1547 & $9.57^{* * *}$ & 1260 & $23.00^{* * *}$ \\
\hline OriginFpl & 2 & 12392 & $28.93^{* * *}$ & 30 & 2.08 & 60167 & 1.47 & 435 & $4.55^{*}$ & 1136 & $7.02^{*}$ & 443 & $8.08^{* *}$ \\
\hline ctype $\times$ OriginFpl & 4 & 6772 & $7.90^{* * *}$ & 43 & 1.47 & 96513 & 1.18 & 289 & 1.51 & 161 & 0.50 & 102 & 0.93 \\
\hline Fam $($ ctype $\times$ OriginFpl $)$ & 400 & 88231 & $5.68^{* * *}$ & 2958 & $1.56^{* * *}$ & 8269727 & $1.26^{*}$ & 19270 & $1.28^{* *}$ & 32750 & $1.97^{* * *}$ & 11085 & $2.02^{* * *}$ \\
\hline Rep & 1 & 4202 & $108.71^{* * *}$ & 32 & $6.79^{* *}$ & 11459 & 0.70 & 781 & $20.80^{* * *}$ & 2520 & $60.80^{* * *}$ & 3203 & $234.11^{*}$ \\
\hline Error & 383 & 14879 & & 1843 & & 6394752 & & 14681 & & 16288 & & 5390 & \\
\hline$R^{2}$ & & & 88 & & .63 & & & & 62 & & 70 & & .75 \\
\hline
\end{tabular}

Abbreviations: GWP, grain weight per plant; KNP, kernel number per plant; KNS, kernel number per spike; PH, plant height; SNP, spike number per plant; TKW, thousand-kernel weight.

${ }^{*} P$-value $<0.05,{ }^{* *} P$-value $<0.01$ and ${ }^{* * *} P$-value $<0.0001$

Table 4 Phenotypic least-square means values for the cross type effect (SELF, WPC and BPC) in model (2)

\begin{tabular}{lllllrl}
\hline Cross types & PH & SNP & KNP & GWP & KNS & TKW \\
\hline SELF & $109.30(\mathrm{a})$ & $10.91(\mathrm{a})$ & $465.87(\mathrm{a})$ & $20.99(\mathrm{a})$ & $42.36(\mathrm{a})$ & $44.74(\mathrm{a})$ \\
WPC & $112.76(\mathrm{ab})$ & $11.47(\mathrm{ab})$ & $528.95(\mathrm{~b})$ & $25.12(\mathrm{~b})$ & $45.58(\mathrm{~b})$ & $47.28(\mathrm{~b})$ \\
BPC & $113.27(\mathrm{~b})$ & $11.60(\mathrm{~b})$ & $533.93(\mathrm{~b})$ & $25.54(\mathrm{~b})$ & $45.56(\mathrm{~b})$ & $47.76(\mathrm{~b})$ \\
\hline
\end{tabular}

Abbreviations: BPC, between-population cross; GWP, grain weight per plant; KNP, kernel number per plant; KNS, kernel number per spike; $\mathrm{PH}$, plant height; SELF, SELFing; SNP, spike number per plant; TKW, thousand-kernel weight; WPC, within-population cross. Letters beside values correspond to multiple comparisons between the cross types.

Table 5 Phenotypic least-square mean values for the origin of the female plant (Le Moulon, Rennes and Toulouse) in model (2)

\begin{tabular}{|c|c|c|c|c|c|c|}
\hline Female plant origin & PH & SNP & KNP & $G W P$ & KNS & $T K W$ \\
\hline Le Moulon & 109.34 (a) & 11.11 (a) & 505.67 (a) & 23.44 (a) & 45.12 (a) & 45.83 (a) \\
\hline Rennes & $117.69(b)$ & 11.26 (a) & 522.18 (a) & $24.98(b)$ & 45.60 (a) & 47.69 (b) \\
\hline Toulouse & 108.30 (a) & 11.61 (a) & 500.91 (a) & 23.31 (a) & $42.78(b)$ & 46.27 (a) \\
\hline
\end{tabular}

Abbreviations: GWP, grain weight per plant; KNP, kernel number per plant; KNS, kernel number per spike; PH, plant height; SNP, spike number per plant; TKW, thousand-kernel weight.

Letters beside values correspond to multiple comparisons between the origins of the female plants.

respectively). Within-population heterosis was always low but significant for $\mathrm{PH}(0.03$ for the three populations), KNS (0.04-0.08, not significant for the Rennes population) and TKW (0.04-0.06). In general, withinpopulation heterosis for the different traits varied in the same proportions when changing from one population to another, except for heterosis for SNP that was marginally negative in the Le Moulon population and between 0.08 and 0.10 in the two other populations. Between-population heterosis was always low or negative whatever the populations and traits and never significant. There was slight outbreeding depression for SNP, KNS, KNP and GWP for the progeny of the cross between the Le Moulon and Rennes populations. Progeny from the Le Moulon and Toulouse crosses also showed weak outbreeding depression for KNS.

\section{Discussion}

Finding rules to apply when designing genetic rescue for threatened fragmented populations is a major issue in conservation biology (Tallmon et al., 2004; Edmands, 2007). In this study we used recently diverged experimental populations to which we applied self-pollination, within- and between-population crosses to investigate the link between the genetic structure of the subdivided population, and fitness-related traits values in the firstgeneration offspring, by assessing within-population heterosis or inbreeding depression, between-population heterosis or genetic load and outbreeding depression. This approach is complementary and intermediate to analytical and simulation studies (Theodorou and Couvet, 2002; Glemin et al., 2003; Jaquiéry et al., 2009) and experimental crosses in natural populations (Fenster and Galloway, 2000; Newman and Tallmon, 2001; Willi et al., 2005) because much information on the history of the populations (time since divergence, population census sizes, initial diversity and so on) is available.

We found high and significant inbreeding depression within populations for four of six traits with systematic superiority of within-population cross progeny compared with selfed progeny. Moreover, little betweenpopulation heterosis was found, with similar values for offspring from between-population and within-population crosses.

\section{Within-population heterosis}

Wheat is a mainly selfing species with an outcrossing rate between 2 and 10\% (Martin, 1990; Enjalbert et al., 1998; Enjalbert and David, 2000). In a selfing species, a high level of homozygosity is expected at all loci. 

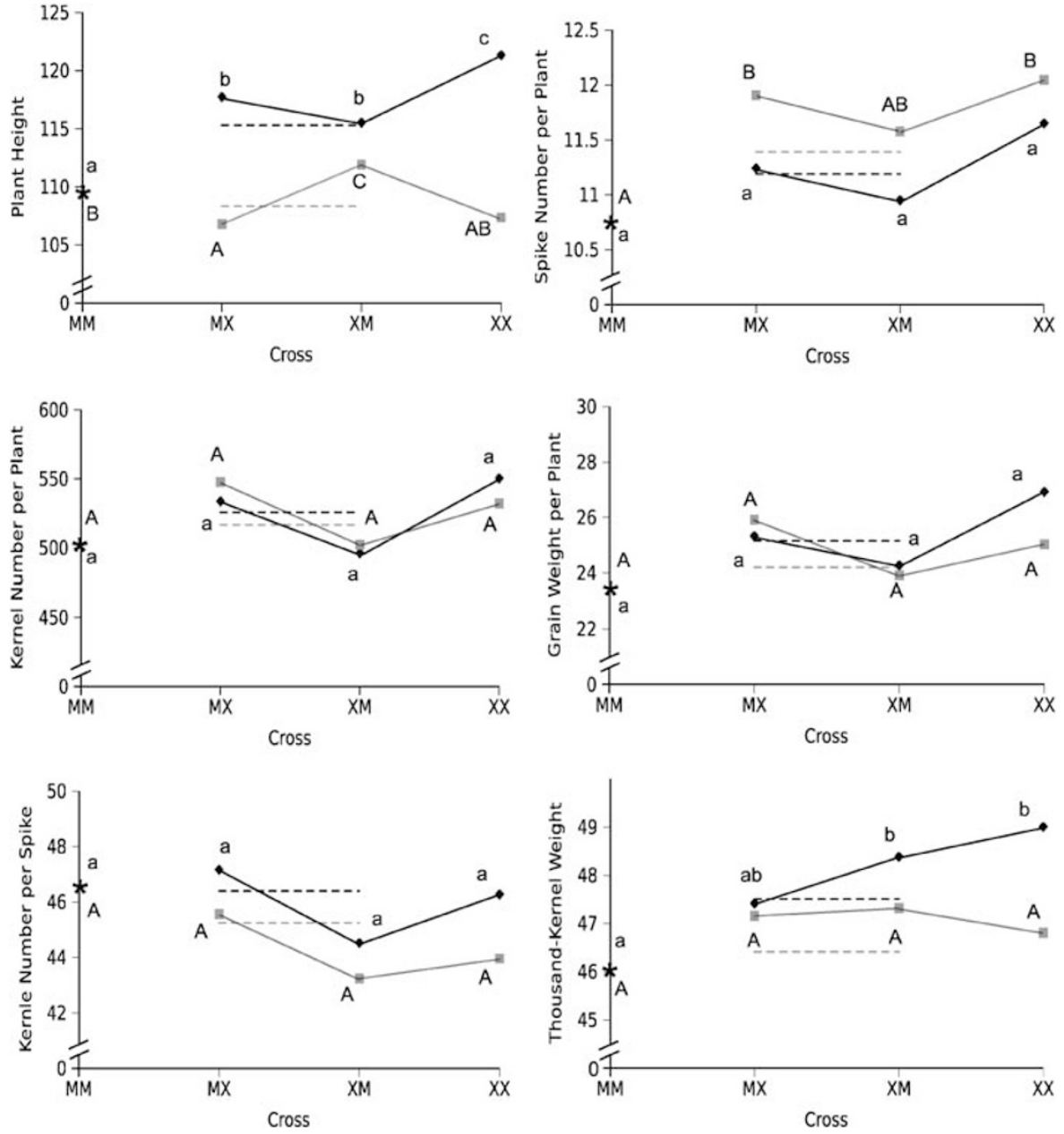

Figure 2 Least-square means values for the cross effect in model (4) for resident within-population crosses (Le Moulon $\times$ Le Moulon (MM)) migrant within-population crosses (XX, $\mathrm{X}$ is Rennes or Toulouse) and between-population crosses (MX for crosses with the female plant from the Le Moulon population and XM for the reciprocal crosses). Black lines represent X parent from the Rennes population, and grey lines represent $X$ parent from the Toulouse population. Horizontal dotted lines represent the mid-parent values between each couple MM and XX. Letters correspond to multiple comparisons between migrant, resident and BPC, lowercase for $\mathrm{X}=$ Rennes and uppercase for $\mathrm{X}=$ Toulouse.

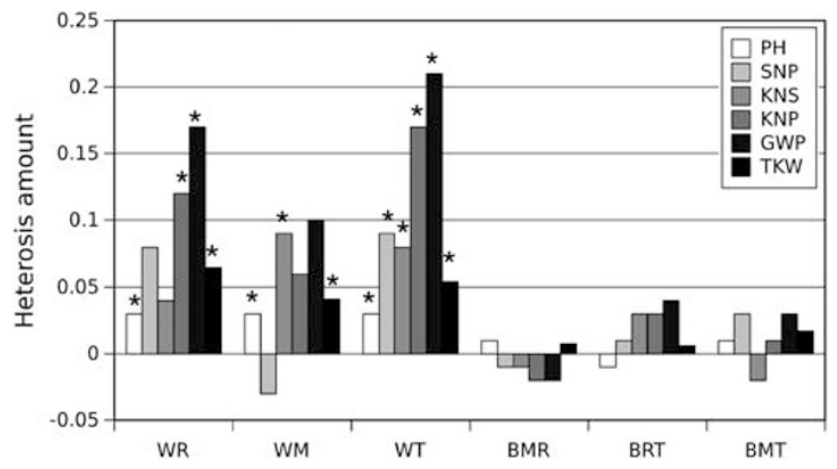

Figure 3 Bar chart of levels of within- and between-population heterosis for quantitative traits. GWP, grain weight per plant; KNP, kernel number per plant; KNS, kernel number per spike; PH, plant height; SNP, spike number per plant; TKW, thousand kernel weight. Heterosis level estimates are as follows: BMR, between Le Moulon and Rennes; BMT, between Le Moulon and Toulouse; BRT, between Rennes and Toulouse; WM, within Le Moulon; WR, within Rennes; WT, within Toulouse.

Recessive deleterious mutations are thus expressed, and selection is expected to purge them efficiently, leading to low inbreeding depression in a selfing population.
Unexpectedly, significant inbreeding depression was revealed in each DM population for four of the six studied traits by comparing selfing and within-population cross progeny. Corresponding within-population cross progeny heterosis values ranged between 4 and $21 \%$. These values are surprisingly higher than the reported values for wheat species: Dreisigacker et al. (2005) found heterosis values for grain yield ranging from -15.33 to $14.13 \%$ with a mean of $0.5 \%$ for crosses between specific inbred lines from a breeding program, whereas Husband and Schemske (1996) estimated that the mean within-population heterosis for 11 selfing species is $5 \%$ for seed production. In fact, inbreeding depression can be divided into two parts: one contributed by lethal or sublethal mutations that will be purged by inbreeding, independent of population size, unless the mutation is totally recessive (Nei, 1968; Bataillon and Kirkpatrick, 2000), and the second because of mildly deleterious mutations that are only partially recessive. This second type will be greatly sensitive to population size and it will decrease as population size becomes smaller and genetic drift increases (Bataillon and Kirkpatrick, 2000).

The evidence of inbreeding depression that we found within each population, associated with a supposedly 
low genetic load, was consistent with the theoretical results of Bataillon and Kirkpatrick (2000) for effective sizes of approximately 100 and deleterious effects of mutations $>0.05$. The researchers showed that small populations $(N \ll 100)$ do not efficiently purge their genetic load, and have an overall reduction in fitness because of the accumulation of deleterious mutations. However, inbreeding depression is not visible when comparing crosses between individuals within these populations to self-fertilized individuals because of the fixation of many loci within the population as a whole. On the contrary, larger populations $(N \gg 100)$ more efficiently purge their genetic load, and allelic diversity within the population is high enough to allow the detection of inbreeding depression when comparing selfed individuals to crosses between individuals within the population.

However, in our case, the two smallest populations, Rennes and Toulouse, showed stronger inbreeding depression than the largest population (Le Moulon). This discrepancy between theoretical expectations from Bataillon and Kirkpatrick (2000) and our experimental results could be explained by the difference of time scale taken into account in the two approaches. Their model assumes that the studied population is at mutationselection-drift equilibrium. After 12 generations, we can hardly assume that DM populations reached such equilibrium. Simple simulations allowed us to assess the probability that a mildly deleterious mutation (mutation effect between 0.01 and 0.10 ) initially existing in the ancestral population would be eliminated after 12 generations and its frequency if instead of being eliminated it remained in the segregating population (see details in the Supplementary Information). For the three populations, the probability of fixation of the deleterious mutation is null (Supplementary Figure S1). The more probable case is that the deleterious mutation could still be segregating in the populations. The expected frequency of the deleterious mutation is lower in the Le Moulon population than in the two others (Supplementary Figure S2), which could explain the lower values of within-population heterosis in this population.

Between-population heterosis or outbreeding depression Studying the first-generation progeny of crosses between individuals from two populations can reveal two mechanisms linked to the subdivided population structure. First, hybridization between divergent populations may result in loss of fitness, known as outbreeding depression. This decline in fitness can be attributed to the effect of locally maladapted genes, which have been selected in another environment (the ecological mechanism) (Waser, 1993) or to genetic mechanisms such as the break-up of positive epistatic interactions or underdominance effects (Lynch, 1991). Second, masking mildly deleterious mutations differentially fixed in the different populations (genetic load at the within-population level) may lead to between-population heterosis (Fenster and Galloway, 2000).

In our study, between-population progeny were never significantly different from within-population progeny, and heterosis at the between-population level was low $(<4 \%)$. This may indicate either both low between- population heterosis and little outbreeding depression or compensation between the two phenomena.

Effective population size is expected to strongly influence the accumulation of a genetic load in a population, as small population sizes will increase genetic drift thus increasing fixation of deleterious alleles (Kimura et al. 1963). Simultaneously small population sizes will cause allele frequency variations among populations, leading to strong genetic differentiation. Hence, crosses between smaller populations are expected to show more heterosis than crosses between larger populations (Whitlock, 2000). On the other hand, larger divergence between populations might lead to outbreeding depression because of genetic incompatibilities (Escobar et al., 2008). Finally, selection is expected to be more efficient in larger populations, when genetic drift is limited, allowing for local adaptation to develop in the population. Hence, crosses between large but locally adapted populations might also yield outbreeding depression. In this study, genetic effective sizes of the populations were relatively large (Table 1) compared with those of wild populations (Frankham, 1995, 1999; Siol et al., 2007) and the $\mathrm{F}_{\mathrm{ST}}$ estimated on 20 neutral markers between the three experimental wheat populations were quite low $\left(\mathrm{F}_{\mathrm{ST}} \approx 7 \%\right.$ ), which was consistent with reduced between-population heterosis. The slightly higher heterosis between the Rennes and Toulouse populations might be because of their larger differentiation $\left(\mathrm{F}_{\mathrm{ST}}=0.10\right)$ and lower $N_{\mathrm{e}}$ (Table 1$)$.

We found high and significant $Q_{\mathrm{ST}}$ values, associated with $Q_{\mathrm{ST}} / \mathrm{F}_{\mathrm{ST}}$ ratios $>1$ for $\mathrm{PH}, \mathrm{KNS}$ and GWP (Table 2). This can be interpreted as the consequence of divergent selection acting on these traits (Whitlock, 1999) as was shown by previous studies on these populations (Rhoné, 2008) and for other traits such as disease resistance (Paillard et al., 2000) or heading date (Goldringer et al., 2006). Non-additive effects such as dominance or epistasis are expected to lower the estimation of $Q_{\mathrm{ST}}$ (Whitlock, 1999; Goudet and Buchi, 2006). Hence, $Q_{\mathrm{ST}} /$ $\mathrm{F}_{\mathrm{ST}}$ ratio of $>1$ is a conservative indication that, in addition to drift, the main force acting on the DM populations is divergent selection, potentially leading to local adaptation of populations in each contrasted site. Comparison of least-square means of the population origins estimated on selfed progeny gave indications of the differentiation at quantitative traits under divergent selection. For GWP and KNS, which are expected to be tightly correlated with fitness, there were no significant differences between Le Moulon and Rennes progeny. However, Toulouse progeny showed significantly lower values for these fitness-related traits, possibly indicating maladaptation of this population to Le Moulon environment. One possible explanation is that the environments of Le Moulon and Rennes are more similar and hence genes selected for local adaptation in Rennes are also adapted to Le Moulon, whereas locally adapted genes selected in Toulouse are maladapted in Le Moulon. Such a pattern is consistent with adaptation to climatic conditions, as climatic conditions of the two northern sites (Le Moulon and Rennes) are quite similar to each other, whereas very different from conditions in the southern site (Toulouse). We studied an aspect of local adaptation by considering that WPC progeny from the Le Moulon population were residents and WPC progeny from the Rennes and Toulouse populations were 
migrants (see Figure 2). Results were not the same as for the analysis of selfed progeny except for PH and KNP. Indeed, there were no differences between populations for GWP and KNS (traits with a significant $Q_{\mathrm{ST}}$ ), whereas there were significant differences for SNP and TKW (traits without significant $Q_{\mathrm{ST}}$ ). This discrepancy could be explained by the fact that phenotypic values are expressions of genetic interactions between male and female alleles in progeny of crosses in addition to simple effect of homozygous female effects as in selfed progeny. This draws attention to the fact that estimation of $Q_{\mathrm{ST}}$ is dependent on the type of family used. Goudet and Buchi (2006) showed that progeny from selfpollination is the most efficient design to estimate $Q_{\mathrm{ST}}$ in the case of self-fertilizing species. Progeny from the Rennes populations showed significantly higher phenotypic values than progeny from the Le Moulon and Toulouse populations for SNP and TKW. This might indicate that the progeny from the Rennes population were more adapted than those from the Le Moulon population. However, differentiated traits for progeny of crosses (SNP and TKW) are less correlated with fitness than GWP because only part of the fitness components are taken into account, whereas GWP integrates both the number of offspring and survival potential of the offspring (heavier seeds are expected to be more viable). SNP and TKW could be therefore taken to represent life history traits and differences rather than different life history strategies.

In addition, the greater plant height of the Rennes progeny in both cases, selfed or progeny of crosses, might give them a competitive advantage when grown side by side with shorter families, even in the case of the 'local' families from Le Moulon.

Overall, knowing that the experimental design was not optimal to precisely assess local adaptation, we found only slight indications of local adaptation for the three populations, which was consistent with limited outbreeding depression. Finally, the most likely explanation is that both between-population heterosis and outbreeding depression were low in the DM system after 12 generations of divergent evolution.

\section{Optimal crossing distance}

A balance of the effects of heterosis and outbreeding depression as a function of relatedness between crossed individuals is expected to lead to the existence of an optimal genetic distance for genetic rescue strategies (for example, in Willi et al., 2005 and reviewed in Tallmon et al., 2004). In this study, we found no clear relationship (neither linear nor quadratic) between the kinship coefficient for pairs of crossed individuals and progeny phenotypic values. In fact, kinship coefficients averaged for each type of cross were very similar, although high values of kinship were slightly more frequent for withinpopulation pairs of individuals (see Supplementary Figure S3). This result shows that neutral diversity is not as informative as desired for assessing potentially adaptive diversity in the DM system, although linkage disequilibrium is expected to be high in a selfing species (Golding and Strobeck, 1979). However, low linkage disequilibrium in DM has been shown around genes submitted to high selective pressure (Raquin et al., 2008).
Conclusion

On the basis of our results from between-population cross progeny, we think that planned migrations between populations would not bring high heterosis in the DM system, thus reducing its possible positive effect. According to Jaquiéry et al. (2009), information from neutral markers indicating that within-population diversity is still at a sufficient level is a clue that there are no important viability problems within the populations. Neutral marker information did not provide relevant predictive information on phenotypic traits and thus on the evolutionary potential of the populations. This might be a general feature to many experimental crossing experiments. Indeed, complementary information about the subdivided population (for example, presence of local adaptation) is necessary to have a more precise picture of the mechanisms involved in the population under consideration.

Lynch (1991) raised the fact that although heterosis may occur in the first generation after crosses, recombination between parental chromosomes and the break-up of coadapted gene complexes could lead to increased outbreeding depression in the following generations. Genetic rescue effects should then be studied beyond the first generation and these studies are currently in progress with the DM wheat populations. In addition, to further study local adaptation in the DM system, cross progeny have to be studied in each site of parental origin to assess the effects of adaptive genes in the environment in which they have been selected.

Local adaptation could be an important mechanism and is found to be quite frequent in natural populations (Merila and Crnokrak, 2001; McKay and Latta, 2002; Hufford and Mazer, 2003). Only very few theoretical studies take into account local adaptation through an environmental gradient along a species range (Garcia-Ramos and Kirkpatrick, 1997; Alleaume-Benharira et al., 2006). Integrating this kind of selection in theoretical or simulation approaches could be very useful to help conservation biologists to make management decisions.

\section{Conflict of interest}

The authors declare no conflict of interest.

\section{Acknowledgements}

We are grateful to the anonymous reviewers for their constructive and detailed comments. We thank J Enjalbert for insightful discussions, providing the simulation program and careful reading of the paper, J Dawson for relevant comments and English correction of the paper and $\mathrm{O}$ Ronce and $\mathrm{S}$ Glémin for relevant suggestions during this work. YR was funded by a grant from the MENRT. This work has been completed despite the obstacles created due to the French research and University organizational reforms and despite the time and effort devoted to resisting them. This resistance is crucial for keeping the public service of research and university teaching alive in France. 


\section{References}

Alleaume-Benharira M, Pen IR, Ronce O (2006). Geographical patterns of adaptation within a species' range: interactions between drift and gene flow. I Evol Biol 19: 203-215.

Bataillon T, Kirkpatrick M (2000). Inbreeding depression due to mildly deleterious mutations in finite populations: size does matter. Genet Res 75: 75-81.

Belkhir K, Borsa P, Chikhi L, Raufaste N, Bonhomme F (2000). GENETIX 4.02, logiciel sous Windows TM pour la génétique des populations. Laboratoire Génome, Populations, Interactions, CNRS UMR 5000.

Bossuyt B (2007). Genetic rescue in an isolated metapopulation of a naturally fragmented plant species, Parnassia palustris. Conserv Biol 21: 832-841.

Dreisigacker S, Melchinger AE, Zhang P, Ammar K, Flachenecker C, Hoisington D et al. (2005). Hybrid performance and heterosis in spring bread wheat, and their relations to SSRbased genetic distances and coefficients of parentage. Euphytica 144: 51-59.

Edmands S (2007). Between a rock and a hard place: evaluating the relative risks of inbreeding and outbreeding for conservation and management. Mol Ecol 16: 463-475.

Enjalbert J, David JL (2000). Inferring recent outcrossing rates using multilocus individual heterozygosity application to evolving wheat populations. Genetics 156: 1973-1982.

Enjalbert J, Goldringer I, David J, Brabant P (1998). The relevance of outcrossing for the dynamic management of genetic resources in predominantly selfing Triticum aestivum L. (bread wheat). Genet Sel Evol 30: 197-211.

Escobar JS, Nicot A, David P (2008). The different sources of variation in inbreeding depression, heterosis and outbreeding depression in a metapopulation of Physa acuta. Genetics 180: 1593-1608.

Fenster C B, Galloway L F (2000). Inbreeding and outbreeding depression in natural populations of Chamaecrista fasciculata (Fabaceae). Conserv Biol 14: 1406-1412.

Fischer M, Matthies D (1997). Mating structure and inbreeding and outbreeding depression in the rare plant Gentianella germanica (Gentianaceae). Am I Bot 84: 1685.

Frankham R (1999). Quantitative genetics in conservation biology. Genet Res 74: 237-244.

Frankham R (1995). Effective population size/adult population size ratios in wildlife: a review. Genet Res 66: 95-107.

Galloway LF, Etterson JR (2005). Population differentiation and hybrid success in Campanula americana: geography and genome size. J Evol Biol 18: 81-89.

Garcia-Ramos G, Kirkpatrick M (1997). Genetic models of adaptation and gene flow in peripheral populations. Evolution 51: 21-28.

Glemin S, Ronfort J, Bataillon T (2003). Patterns of inbreeding depression and architecture of the load in subdivided populations. Genetics 165: 2193-2212.

Golding GB, Strobeck C (1979). Linkage disequilibrium in a finite population that is partially selfing. Genetics 94 : 777-789.

Goldringer I, Brabant P, Gallais A (1997). Estimation of additive and epistatic genetic variances for agronomic traits in a population of doubled-haploid lines of wheat. Heredity 79: 60-71.

Goldringer I, Enjalbert J, David J, Paillard S, Pham JL, Brabant P (2001). Dynamic management of genetic resources: a 13-year experiment on wheat. In: Cooper $\mathrm{H}$, Spillane $\mathrm{C}$, Hodgkin $\mathrm{T}$ (eds). Broadening the Genetic Base of Crop Production. CABI Publishing: Wallingford, UK, pp 245-269.

Goldringer I, Prouin C, Rousset M, Galic N, Bonnin I (2006). Rapid differentiation of experimental populations of wheat for heading time in response to local climatic conditions. Ann Bot 98: 805-817.

Goudet J, Buchi L (2006). The effects of dominance, regular inbreeding and sampling design on $Q_{\mathrm{ST}}$, an estimator of population differentiation for quantitative traits. Genetics 172: 1337-1347.

Greeff JM, Van Vuuren GJJ, Kryger P, Moore JC (2009). Outbreeding and possibly inbreeding depression in a pollinating fig wasp with a mixed mating system. Heredity 102: 349-356.

Guyomarc'h H, Sourdille P, Charmet G, Edwards K, Bernard M (2002). Characterisation of polymorphic microsatellite markers from Aegilops tauschii and transferability to the D-genome of bread wheat. Theor Appl Genet 104: 1164-1172.

Hardy OJ, Vekemans X (2002). SPAGeDi: a versatile computer program to analyse spatial genetic structure at the individual or population levels. Mol Ecol Notes 2: 618-620.

Henry JP, Pontis C, David J, Gouyon PH (1991). An experiment on dynamic conservation of genetic resources with metapopulations. In: Seitz A, Loeschke V (eds). Species Conservation: A Population Approach. Birkhaüser Verlag: Basel, pp 185-198.

Houle D (1992). Comparing evolvability and variability of quantitative traits. Genetics 130: 195-204.

Hufford KM, Mazer SJ (2003). Plant ecotypes: genetic differentiation in the age of ecological restoration. Trends Ecol Evol 18: $147-155$.

Husband BC, Schemske DW (1996). Evolution of the magnitude and timing of inbreeding depression in plants. Evolution 50: $54-70$.

Jaquiéry J, Guillaume F, Perrin N (2009). Predicting the deleterious effects of mutation load in fragmented populations. Conserv Biol 23: 207-218.

Jost L (2008). GST and its relatives do not measure differentiation. Mol Ecol 17: 4015-4026.

Keller LF, Waller DM (2002). Inbreeding effects in wild populations. Trends Ecol Evol 17: 230-241.

Kimura M, Maruyama T, Crow JF (1963). The mutation load in small populations. Genetics 48: 1303-1312.

Loiselle BA, Sork VL, Nason J, Graham C (1995). Spatial genetic structure of a tropical understory shrub, Psychotria officinalis (Rubiaceae). Am J Bot 82: 1420.

Lynch M (1991). The genetic interpretation of inbreeding depression and outbreeding depression. Evolution 45: 622-629.

Lynch M, Conery J, Burger R (1995). Mutation accumulation and the extinction of small populations. Am Nat 146: 489-518.

Madsen T, Shine R, Olsson M, Wittzell H (1999). Conservation biology: restoration of an inbred adder population. Nature 402: 34-35.

Martin TJ (1990). Outcrossing in twelve hard red winter wheat cultivars. Crop Sci 30: 59-62.

McKay JK, Latta RG (2002). Adaptive population divergence: markers, QTL and traits. Trends Ecol Evol 17: 285-291.

Merila J, Crnokrak P (2001). Comparison of genetic differentiation at marker loci and quantitative traits. J Evol Biol 14: 892-903.

Nei M (1987). Molecular Evolutionary Genetics. Columbia University Press: New York, NY, USA.

Nei M (1968). The frequency distribution of lethal chromosomes in finite populations. Proc Natl Acad Sci USA 60: 517-524.

Nei M, Tajima F (1981). DNA polymorphism detectable by restriction endonucleases. Genetics 97: 145-163.

Newman D, Tallmon DA (2001). Experimental evidence for beneficial fitness effects of gene flow in recently isolated populations. Conserv Biol 15: 1054-1063.

Paillard S, Goldringer I, Enjalbert J, Doussinault G, VallavieillePope C, Brabant P (2000). Evolution of resistance against powdery mildew in winter wheat populations conducted under dynamic management. I-Is specific seedling resistance selected? Theor Appl Genet 101: 449-456.

Pollak E (1987). On the theory of partially inbreeding finite populations. I. Partial selfing. Genetics 117: 353-360.

Raquin A, Brabant P, Rhoné B, Balfourier F, Leroy P, Goldringer I (2008). Soft selective sweep near a gene that increases plant height in wheat. Mol Ecol 17: 741-756. 
Rhoné B (2008). Genetic mechanisms involved in climatic adaptation of experimentally evolving populations of bread wheat. Msc Thesis, AgroParisTech, Paris, France.

Rhoné B, Remoué C, Galic N, Goldringer I, Bonnin I (2008). Insight into the genetic bases of climatic adaptation in experimentally evolving wheat populations. Mol Ecol 17: 930-943.

Röder MS, Korzun V, Wendehake K, Plaschke J, Tixier MT, Leroy P et al. (1998). A microsatellite map of wheat. Genetics 149: 2007-2023.

SAS Institute (2000). SAS/STAT user guide, Version 8. SAS Publishing: Cary, NC, USA.

Schierup MH, Christiansen FB (1996). Inbreeding depression and outbreeding depression in plants. Heredity 77: 461-468.

Siol M, Bonnin I, Olivieri I, Properi JM, Ronfort J (2007). Effective population size associated with self-fertilization: lessons from temporal changes in allele frequencies in the selfing annual Medicago truncatula. J Evol Biol 20: 2349-2360.

Somers DJ, Isaac P, Edwards K (2004). A high-density microsatellite consensus map for bread wheat (Triticum aestivum L.). Theor Appl Genet 109: 1105-1114.

Spitze K (1993). Population Structure in Daphnia obtusa: quantitative genetic and allozymic variation. Genetics 135: 367-374.

Tallmon DA, Luikart G, Waples RS (2004). The alluring simplicity and complex reality of genetic rescue. Trends Ecol Evol 19: 489-496.

Theodorou K, Couvet D (2002). Inbreeding depression and heterosis in a subdivided population: influence of the mating system. Genet Res 80: 107-116.

Theodorou K, Couvet D (2006). Genetic load in subdivided populations: interactions between the migration rate, the size and the number of subpopulations. Heredity 96: 69-78.
Vila C (2003). Rescue of a severely bottlenecked wolf (Canis lupus) population by a single immigrant. Proc R Soc B Biol Sci 270: 91-97.

Wang J (2004). Application of the one-migrant-per-generation rule to conservation and management. Conserv Biol 18: 332-343.

Waples RS (1989). A generalized approach for estimating effective population size from temporal changes in allele frequency. Genetics 121: 379-391.

Waser NM (1993). Population structure, optimal outbreeding, and assortative mating in angiosperms. In: Thornhill NW (ed). The Natural History of Inbreeding and Outbreeding: Theoretical and Empirical Perspectives. University of Chicago Press: Chicago, pp 173-199.

Waser NM, Price MV, Shaw RG (2000). Outbreeding depression varies among cohorts of Ipomopsis aggregata planted in nature. Evolution 54: 485-491.

Weir B, Cockerham C (1984). Estimating F-statistics for the analysis of population structure. Evolution 38: 1358-1370.

Whitlock MC (1999). Neutral additive genetic variance in a metapopulation. Genet Res 74: 215-221.

Whitlock MC (2000). Local drift load and the heterosis of interconnected populations. Heredity 84: 452-457.

Willi Y, Fischer M (2005). Genetic rescue in interconnected populations of small and large size of the self-incompatible Ranunculus reptans. Heredity 95: 437-443.

Willi Y, Van Buskirk J, Fischer M (2005). A threefold genetic allee effect population size affects cross-compatibility, inbreeding depression and drift load in the self-incompatible ranunculus reptans. Genetics 169: 2255-2265.

Wright S (1969). Evolution and the Genetics of Populations. Vol. 2. The Theory of Gene Frequencies. University of Chicago Press: Chicago.

Supplementary Information accompanies the paper on Heredity website (http://www.nature.com/hdy) 